\title{
National benchmarks for treatment of seniors' mental health recommended
}

$\mathrm{M}$ inimum benchmarks should be implemented regarding the number of beds and health care workers available to treat seniors with mental health problems, the Mental Health Commission of Canada recommends.

The benchmarks, such as the provision of a minimum 7.5 residential mental health beds per 10000 elderly, would serve to reduce the current fragmentation of mental health services for seniors, the nonprofit organization argued in its Guidelines for Comprehensive Mental Health Services for Older Adults in Canada (www.mentalhealth commission.ca/seniorsguidelines/seniors -guidelines.pdf).

As Canada's population ages, it also becomes more susceptible to mental health problems and illness, says Dr. Marie-France TourignyRivard, chair of the commission's Seniors Advisory Committee. A quarter of older Canadians already suffer from mental health problems, as compared to a fifth of the general population, yet mental health services for seniors are frequently difficult to access and navigate, she says.

In addition to a minimum 7.5 residential beds for longer-term stabilization and treatment for seniors with severe and persistent behavioural and psychological symptoms of dementia in a specially designed unit in a long-term care facility, the guidelines recommend, per 10000 elderly:

- 5.5 full-time equivalent (FTE) health professionals for seniors' mental health outreach and community teams to provide "consultation-liaison services to residential (long-term) care homes, collaborative/shared care in community settings and capacity building models of care"

- 5.25 FTE health professionals for intensive community treatment and support for seniors with persistent and severe mental illnesses

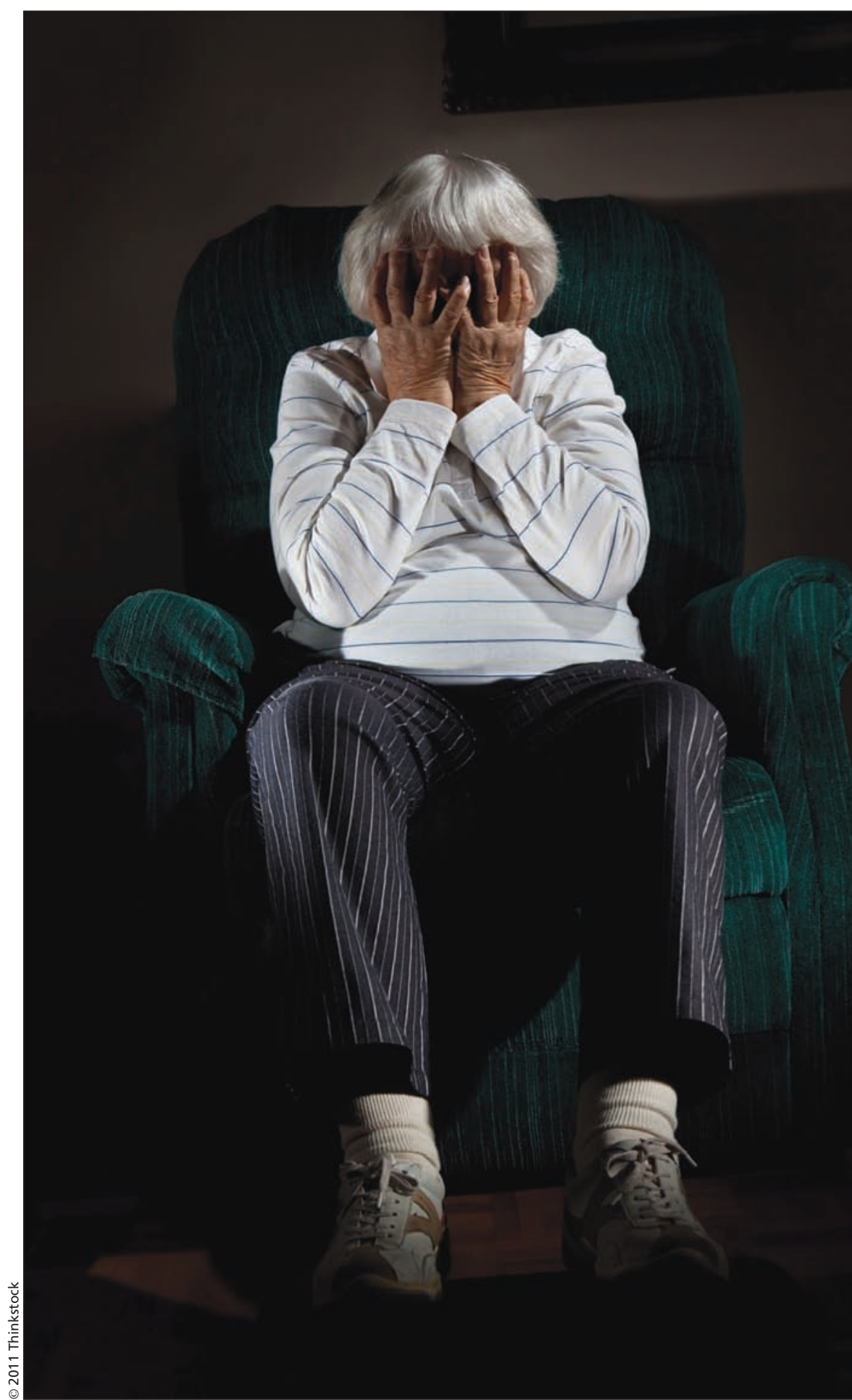

Accurate diagnosis of mental illness in seniors can be challenging because many older adults live with multiple chronic health conditions. 
- 3 acute short-term inpatient psychiatric beds located on a general acute care psychiatric inpatient services, "ideally with geriatric psychiatrist consultation/support and average length of stay of approximately 1 month"

- 3.3 specialized geriatric psychiatry inpatient beds for assessment and active treatment for seniors who require the care of a specialized geriatric team in hospital, with an average length of stay below 90 days

- 3 or fewer specialized geriatric psychiatry inpatient beds, depending on the availability of residential mental health beds, for rehabilitation or chronic care in a psychiatric hospital over 90 days for those with severe and persistent disorders.

To date, "no region in Canada has met these benchmarks 100\%," Rivard says, adding that most areas have some of the core elements in place that can readily be strengthened.

The commission recommended that minimum guidelines be used to "review existing services and staffing levels and guide future allocation and deployment of resources, taking into account the size of the population, existing gaps in services and bottlenecks, as well as priorities of the community."

The commission, which was established in 2007 by the Conservative government and provided $\$ 140$ million over 10 years to craft a national mental health strategy, argued that the benchmarks and guidelines for the treatment of seniors with mental health problems would help to create a system where "mental health is everybody's business."

To that end, the guidelines recommended that:

- "Mental health promotion should be embedded in all policies, programs, and services for all older adults (including those with mental illness) and their caregivers, and encompass anti-stigma strategies, public awareness, education, and training;

- Older adults, caregivers, service providers and the public should be informed about the importance of early identification of symptoms of mental illness, prevention strategies and the hope for recovery and well-being;

- The specific components of an integrated mental health service system for older adults may vary according to local context and resources, but all service systems should provide access to the following range of services for the entire senior population: community-based support services, primary care services, general mental health services and specialized seniors mental health services (including specialized community and outreach services to residential care facilities as well as specialized geriatric psychiatry inpatient services)."

In arguing that the system become more "recovery oriented," the commission also noted that implementation of the guidelines would require "crosssectoral and multi-sectoral partnerships/collaborations that go beyond/ outside the mental health care/service system ... (and) clear mechanisms in place to facilitate collaboration and access between services."

While noting that many older adults live with multiple chronic health conditions, which can make accurate diagnosis more challenging, Rivard says the system simply must become more effective in diagnosing mental illness in seniors.

To that end, the commission urged more training of both health care workers and informal caregivers. "Without proper training and attention, treatable illnesses can, and do, go unnoticed by healthcare providers," the guidelines state. "Transformation of a mental health service system must include training, education and support for caregivers and health care providers to increase their capacity to respond to the mental health needs of seniors."

Meanwhile, family and other informal caregivers, who provide more than $80 \%$ of care to seniors and contribute more than $\$ 5$ billion of unpaid labour to the health care system, "are not adequately supported by health and social services or by public policy," the commission stated. "Most public policies that affect caregivers directly or indirectly (e.g., health services, labour) have been developed without taking into account their needs and may have unintentional negative effects on caregivers and their families, compromising their capacity to provide care." - Lauren Vogel, CMAJ

CMAJ 2011. DOI:10.1503/cmaj.109-4028 